\title{
Children are not like other loads: A cross-cultural perspective on the influence of burdens and companionship on human walking
}

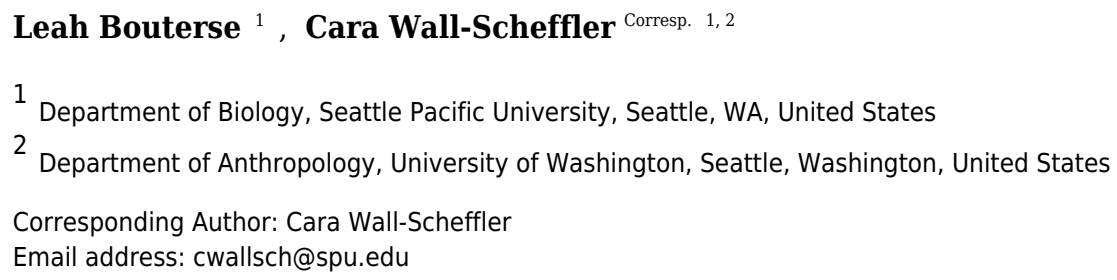

A major portion of humans' activity-based energy expenditure is taken up by locomotion, particularly walking. Walking behaviors have energetic outcomes and as such can be important windows into how populations and groups adjust to different environmental and task constraints. While sex differences in the speed of paired walkers have been established by others, the dynamics of how walkers adjust their speed in more varied groups and in groups containing children remains unexplored. Furthermore, little ecological data exists to illustrate the relationships between walking speed and childcarrying. Here, we aim to determine how culture impacts the effects of group composition and infant-carrying on walking speed. Because the determinants of group dynamics and parental investment are partially cultural, we examine walking behavior in the Northwestern United States and in Central Uganda. Using an observational method, we recorded the speed, load carriage, and group composition of pedestrians in a single naturalistic urban environment within each country. Our data suggest that children are treated fundamentally differently than other loads or the presence of walking partners, and that major speed adjustments are child-dependent. Our data furthermore indicate that Ugandans walk more slowly in groups than when alone, while Americans walk more quickly in groups. Clear distinctions between the groups make large generalizations about walking behavior difficult, and highlight the importance of culturally specific contexts. 
3 Children are not like other loads: A cross-cultural perspective on the influence of burdens and

4 companionship on human walking

5

6

7 Leah Bouterse

8 Department of Biology

9 Seattle Pacific University

$1033073^{\text {rd }}$ Ave West

11 Seattle WA 98119

12

$13 \&$

14

15 Cara Wall-Scheffler*

16 Department of Anthropology

17 University of Washington

18 Box 353100

19 Seattle WA 98195

20

cwallsch@u.washington.edu

22

23

24

25

26

27

28

29

30

31

32

33

34

35

36

37

38

39

40

41

42

+12062812201

Pages: 15

Figures: 3

Tables: $3+1$ Supplemental

*corresponding author 


\section{ABSTRACT}

44

45 A major portion of humans' activity-based energy expenditure is taken up by locomotion,

46 particularly walking. Walking behaviors have energetic outcomes and as such can be important

47 windows into how populations and groups adjust to different environmental and task constraints.

48 While sex differences in the speed of paired walkers have been established by others, the

49 dynamics of how walkers adjust their speed in more varied groups and in groups containing

50 children remains unexplored. Furthermore, little ecological data exists to illustrate the

51 relationships between walking speed and child-carrying. Here, we aim to determine how culture

52 impacts the effects of group composition and infant-carrying on walking speed. Because the

53 determinants of group dynamics and parental investment are partially cultural, we examine

54 walking behavior in the Northwestern United States and in Central Uganda. Using an

55 observational method, we recorded the speed, load carriage, and group composition of

56 pedestrians in a single naturalistic urban environment within each country. Our data suggest that

57 children are treated fundamentally differently than other loads or the presence of walking

58 partners, and that major speed adjustments are child-dependent. Our data furthermore indicate

59 that Ugandans walk more slowly in groups than when alone, while Americans walk more

60 quickly in groups. Clear distinctions between the groups make large generalizations about

61 walking behavior difficult, and highlight the importance of culturally specific contexts.

62

63 


\section{INTRODUCTION}

65

66

67

Today, foraging populations who travel far distances face consistent pressures to decrease the energetic cost of their journeys and even people living in Western contexts clearly make individual speed and gait choices that minimize energy expenditure (1-5). Theoretically, energyminimizing behavior is believed to stem from the overarching principle that time and energy spent on one task (e.g. walking) takes away from energy available for reproduction and in the case of social organisms, investment in social groups (6-8). Optimization of locomotor costs then, significantly impacts feeding, foraging, avoiding predators, and reproducing, as well as sociality (9). The cost of locomotion has been shown to depend on morphological as well as behavioral conditions. For example, increases in body size and limb length are associated with decreased locomotor cost relative to mass $(10,11)$. Additionally, males (i.e. bigger individuals with longer lower limbs) walk at faster speeds than smaller females across different populations $(12,13)$. Behaviors, such as load carrying and walking in groups, also impact the costs and decisions associated with walking and can potentially offer additional selection pressures to single-person free walking $(9,14-16)$.

Like many mammals, human walking shows a speed at which energy expenditure per distance travelled is lower than all other speeds-- as demonstrated by the curvilinear relationship between cost of transport (metabolic cost for a given distance) and speed $(17,18)$. Thus, when walking alone, humans are expected to prefer speeds that minimize costs under particular conditions; however, when walking in groups, humans do not necessarily walk at the speed that minimizes energy. Boles (14) and Costa (12) show speed changes related to group size and composition in an ecological setting. Dyads walk more slowly than individuals, since one member must adjust for the slower speed of their partner (14). Wagnild and Wall-Scheffler (9) 
88 suggest that these adjustments are based on gender and the emotional closeness between walkers.

89 While men and women walking together each adjust their speed away from each's individualized

90 energetic optimum, the same does not hold true for individuals that share a romantic relationship.

91 In heterosexual romantic partners, men will walk more slowly than his energetic optima in order

92 to match the walking speed of his partner. When men walk with other men, their speeds tend to

93 increase $(9,12)$. Social phenomena in some cultures - including a greater degree of social

94 hierarchy in American male groups and American male aversion to showing same-sex intimacy--

95 have been thought to explain this observation (12). The relationships between sex and group

96 walking speed hold true for triads as well as dyads, but the effects have been shown to decrease

97 for groups greater than three individuals (12).

98 Load-bearing is another behavior which impacts walking costs and speed. The net

99 metabolic power of walking has been shown to depend on load mass: data show that a unit of

100 load mass is more expensive to transport than a unit of body mass placed at the same position

101 relative to the carrier's center of mass $(15,19)$. The energetically optimal walking speed for an

102 individual tends to decrease with increasing load mass in multiple studies $(8,15)$. Groups of East

103 African women, though, have been shown to carry loads up to $20 \%$ of their body mass without

104 increased costs, potentially due to some change in kinematics (20). Effectiveness of carrying a

105 load seems to depend on its position. Generally, loads are shown to add less metabolic cost the

106 closer they are carried to the center of mass, and more so if they are carried on the back than on

107 the front $((21,22)$, though see $(8)$ for the low cost of carrying on the front and $(20,23)$ for low

108 costs of carrying on the head).

109 The carriage of children is a special case of load bearing. While regular carriage of heavy

110 food or goods applies primarily to human primates, child loads are common across species. 
111 Particularly for mammalian species, care and transport of offspring during lactation presents

112 enormous energetic costs for one parent, often the mother $(19,24)$. Despite the metabolic costs

113 of carrying infants, it remains a widespread strategy across mammals (25). Amongst primates,

114 infants either "ride" while grasping to parents' fur or are transported orally (24). Ross (24)

115 argues that fur riding has evolved independently and been conserved more than five times in

116 primates. While maternal carrying remains prevalent across primate taxa, many New World

117 Monkey fathers, as well as dominant male baboons, also carry infants (25).

118 Non-human primate infants are typically carried on the mother's back or front, often

119 relying on the infant's grip. In humans, however, carriage relies on the parents without help from

120 the child's grip (25). Though ecological data on human infant-carrying is limited, Rhinegold and

121 Keen (25) found that, in an American urban center, women carried infants more often than men

$122(58.6 \% ; 320 / 546)$, but that older children were more likely carried by men. Though back loads

123 are less metabolically costly than side loads, they observed most infants being carried on the side

124 (21, 25). Side carrying allows accessible interaction between child and parent (26).

125 Humans' speed decisions when walking with others and carrying children provides

126 insight into the adaptive influence of social relationships. Understanding the circumstances under

127 which people deviate from their optimum speeds reveals the energy tradeoffs and social

128 interactions in which people engage. However, the dynamics of how walkers adjust their speed

129 in varied groups and in groups containing children remains unexplored. Furthermore, little

130 ecological data exists to illustrate the relationships between walking speed and child-carrying.

131 Because the determinants of group dynamics and parental investment are partially cultural, the

132 present study examines walking behavior between samples from the Northwestern United States

133 and Central Uganda. Traditional Ugandan culture tends toward communalism and high-contact, 
134 whereas traditional United States culture is individualistic and low-contact. We hypothesize that,

135 across cultures, walking with or carrying children will result in significant decreases in walking

136 speed compared to walking with adults or carrying comparable loads of food or goods.

\section{METHODS}

139 Subjects

140 A total of 1,721 subjects were observed in metropolitan public areas walking alongside

141 roads. 969 subjects (355 male and 614 female) were observed in Central Uganda and 752

142 subjects (337 male and 415 female) were observed in Washington State, U.S. All subjects

143 observed purposefully walking in a steady state toward a destination (i.e. not for exercise) and

144 for whom we could monitor their entire trajectory between the two markers were included.

145 Persons walking at a brisk pace and wearing fitness attire without additional medium to large

146 loads were judged to be walking for exercise. Only subjects who walked uninterrupted through

147 the observation area and of whom the observer had an unobstructed view were included. This

148 sample included persons walking alone or in groups, as well as those walking unloaded or

149 loaded. Subjects reflected a normal range of body sizes, which has been shown to be similar for

150 American and Ugandan samples $(27,28)$. All procedures were approved by Seattle Pacific

151 University’s IRB Committee; IRB \#151606011.

152 Procedure

153 From a removed viewing position, an observer recorded the subject's speed as the time

154 taken to walk between pre-measured stationary markers. We used a stopwatch to measure time

155 between the markers, as this is considered the gold-standard method of monitoring pedestrians

$156(9,29-39)$. The stationary markers measured 7.3m apart in the Ugandan location and 9.7m apart 
157 in the United States location. These distances, as well as the position of the observer, were

158 consistent across all collection days. The observer started the stopwatch when the subject's

159 estimated center of mass crossed the first measured mark, and stopped the stopwatch when it

160 passed the second measured mark. Only people who walked close to the markers were measured,

161 and thus potential problems due to parallax were minimized. Speed in meters per second (m/s)

162 was determined by dividing the marked distance by subject's crossing time.

163 The observer recorded the sex and general age category of the subject (i.e. child,

164 teenager, adult, or older adult; Table 1). Any loads carried or pushed by the subject were

165 categorized by type (i.e. child, food, goods, or stroller), position (i.e. front, back, side, or

166 shoulder), and size (i.e. small, medium, or large) of loads carried by the subject. Strollers, while

167 not carried on the arms or torso like other loads, have been shown to significantly moderate

168 speed and were of interest as an infant-transport device (40). A small load was a purse or a small

169 bag in the hands; a medium load was a torso-sized bag such as a backpack; a large load was an

170 oversized bag (Table 2).

171 When the load carried was a human child, the relative age category (i.e. infant $(<1$ year

172 old), toddler (1-3 years old), or child (>3 years old)) of the child was recorded. These related to

173 the size of other loads with infants being classified as a medium load, and toddler and children

174 being classified as large loads (Table 2). Finally, the observer recorded the gender and age

175 category of all other individuals accompanying the focal walker. When adults were observed

176 walking in groups, the observer chose the group member closest to the markers as the focal

177 figure for data collection (see (41) for synchronous walking speeds between partners). Children

178 were selected as the focal figure only when walking alone or in groups of only children. 
All Ugandan and American subjects were observed at a single viewing position within

180 the respective country. In Uganda, that location was $0^{\circ} 21^{\prime} 38.2^{\prime \prime} \mathrm{N}, 32^{\circ} 44^{\prime} 48.3^{\prime \prime} \mathrm{E}$. This was a

181 walking path along Kampala-Jinja Road in Mukono, Uganda. In the United States, the location

182 was $47^{\circ} 38^{\prime} 18.3^{\prime \prime} \mathrm{N} 122^{\circ} 21^{\prime} 22.9^{\prime \prime} \mathrm{W}$. This was also a walking path (pedestrian sidewalk), located

183 in Seattle, Washington.

184 In both locations, (i.e. the Ugandan and American streets) subjects were observed

185 walking in central "errand running" areas near grocery stores and shopping centers over flat

186 ground. Neither location crossed a crosswalk. Both areas had wide, beside-roadway paths such

187 that subjects could comfortably walk abreast in groups. In the United States, subjects walked on

188 paved concrete, while Ugandan subjects walked on packed, smooth dirt. These conditions were

189 typical of the environment; we chose to match circumstances as closely as possible (that is, style

190 and purpose of walking) which meant we were not able to match substrate perfectly. All data

191 were collected in the early or middle afternoon. Weather conditions during each observation

192 period were also recorded, though did not have any significant influence on any model.

193 Data Analysis

194

Data were analyzed using SPSS Statistics via a univariate general linear model

with speed as the dependent variable. The average temperature during collections times was $21^{\circ}$

$196 \mathrm{C}$ in Uganda and $14^{\circ}$ Celsius in the United States. Weather conditions did not significantly

197 impact walking speeds ( $\mathrm{p}>0.197$ ). Data were split by location (i.e. Ugandan observation site or

198 United States observation site) when analyzing effects within a sample. Walker-gender, type of

199 load carried, group composition, number of group members, and the presence of children in the

200 group were tested as factors against the dependent variable of walking speed. Group composition

201 was coded according to the following categories: alone, only men in the group (including walker 
202 whose speed was being monitored); only women in the group (including walker whose speed 203 was being monitored); only adult men and women; men and children; women and children; men,

204 women, and children; and only children. Because of the small sample of older adult subjects,

205 they were included within the "adults" category. Any category including children accounted for 206 groups in which children walked freely or were carried.

207 Tukey post-hoc tests were done on each factor.

208

209

\section{RESULTS}

210

The effects of the type of load carried, the presence of children, and group composition

211 on walking speed each differ significantly between people walking in Central Uganda and people

212 walking in the West Coast of the United States $(\mathrm{p}<0.001)$. Across all walking groups, Ugandans

213 on average walked $2.6 \%$ faster than Americans $(\mathrm{p}<0.01)$. However, while Ugandans walking

214 alone walked 11.3\% faster than Americans walking alone (Supplemental Table 1), Ugandan

215 walkers in groups were slower than both American groups (18.3\% slower) and individual

216 Ugandans (11.7\% slower) (Fig. 1, p <0.001). In Uganda, speed decreased with group size, while

217 speed increased with group size in America (Fig. 1, p $<0.001$ for both). Sixty-eight percent

218 (663/969) of Ugandans walked alone, as did 73\% (551/752) of Americans (Table 3). Men

219 walked $18 \%$ faster than women in Uganda $(\mathrm{p}<0.01)$, while American men and women walked at 220 about the same speeds $(\mathrm{p}=0.411)$.

221 Load

222 In both locations, women were much more likely to be loaded than were men (Table 3;

$223 \mathrm{p}<0.001)$. Ugandans walked 10\% more slowly when loaded, while Americans walked 3.4\%

224 faster when loaded ( $<<0.001$ for both). This trend held true in both locations for loads of all 
225 sizes. Goods (e.g. purses, backpacks, or items for sale) were the most common load type for both

226 locations, constituting $73 \%(n=1,061 / 1,450)$ of loads carried. In both locations, food and goods,

227 but not children, were carried at similar speeds when controlling for size (Fig. 2; p<0.01).

228 Central Ugandans walked significantly faster when back-loaded than when front loaded

229 (p<0.05). In America, people walked slower when back-loaded (e.g. with a backpack) than when

230 front-loaded $(\mathrm{p}<0.001)$.

231 Children

232 Of the 1,449 people carrying loads, 158 carried children (11\%). The effect of carrying

233 children on walking speed was dependent upon location ( $\mathrm{p}<0.001)$. Ugandans walked between

234 15.4-17.6\% more slowly when carrying children than when carrying food or goods (Fig. 2;

$235 \mathrm{p}<0.01$ ), while Americans walked between 19.8-21.2\% more quickly (Fig. 2; $\mathrm{p}<0.01$ ). These

236 relationships remained significant when child loads were compared to food or good loads of the

237 same size category $(\mathrm{p}<0.01)$. Americans' speed did not differ significantly amongst food, goods

238 or strollers ( $\mathrm{p}>0.05)$. Women $(143 / 158)$ were overall more likely to carry children than were men

$239(15 / 158 ; \mathrm{p}<0.01)$. American children were more often carried on the front $(\mathrm{n}=11 / 15)$ than the

240 side $(n=3 / 15)$ or back $(n=1 / 15)$. Ugandan children were most frequently carried on the front

$241(\mathrm{n}=57 / 143)$ or the side $(\mathrm{n}=56 / 143)$ of the subject. While Ugandan women carried children on

242 their fronts, backs, or sides, Ugandan men never carried children on their backs but only on the

243 front or side. Position of the child load was not significantly correlated to speed in either location $244(\mathrm{p}>0.05)$.

245 Amongst Ugandans, women carried infants $(\mathrm{n}=100 / 133)$ more often than toddlers

$246(n=31 / 133)$ and children $(n=2 / 133)$, while men carried toddlers $(n=7 / 11)$ more often than infants 
$247(\mathrm{n}=4 / 11)$. American women and men did not carry infants at a higher rate than toddlers. Age

248 category of the child-load had no significant relationship to speed $(\mathrm{p}>0.05)$.

249 Whether children were present in the walking group significantly influenced speed

$250(\mathrm{p}<0.001)$; however, once children were present $(\mathrm{n}=304)$, there were no significant differences

251 between whether children were carried, walked by themselves, or were pushed in a stroller $(\mathrm{p}=$

252 0.168). American groups walked faster when children were present, while Ugandan groups

253 walked more slowly when children were present $(\mathrm{p}<0.001)$.

254 Group Composition

255 The effect of group composition on walking speed was highly dependent on the sample

256 location (Fig 3; $<<0.001$ ). Amongst Ugandans, the walking speeds of groups with men, women

257 and children, men-only groups, and groups of children are not significantly different from the

258 walking speeds of individuals alone ( $p>0.267$ ). All other group compositions (see Fig. 3) walk

259 more slowly than people walking alone $(\mathrm{p}<0.05)$, but do not show any significant differences

260 from each other (e.g. groups of men with children walk about the same pace as groups of women

261 with children - which is quite slow). Amongst Americans, groups of only children walked at

262 similar speeds to individuals walking alone ( $\mathrm{p}>0.986)$ while all other group types walked faster

263 than individuals walking alone $(\mathrm{p}<0.01)$. There were no significant differences in speed between

264 any American group types.

265

266

267

268

Sex Differences in Group Walking

While group composition was significantly correlated to speed in both samples $(\mathrm{p}<0.05)$,

there was a significant interaction between walker sex and their group composition only in the

270 Ugandan sample $(\mathrm{p}<0.01)$. In Central Uganda, men alone walk significantly faster than men

271 walking with other men $(\mathrm{p}<0.05)$ while men alone in the West Coast United States walked 
272 significantly slower than men walking with other men $(\mathrm{p}<0.01)$. Ugandan women tended to walk

273 more slowly in groups of women than when alone $(\mathrm{p}=0.10)$, but American women walked faster

274 in groups of women than when alone $(p<0.001)$. Each of these analyses included lone male or

275 female subjects from all age groups.

276

277 DISCUSSION

278

279

In the present study, we observe that Ugandan and American walkers choose different

280 speed strategies based on universal situations - that is, just because child carrying and group

281 walking are human universals, it is clear that there are not universal solutions to these situations!

282 While American walkers tend to increase their speeds when walking in groups, Ugandan walkers

283 decrease their speeds when walking with others. Ugandans alone walked faster than Americans

284 alone, even though all group types of Ugandans walked slower than their American counterparts.

285 These differences in speed decisions also extended to individuals walking alongside or carrying

286 children; Ugandans slow down when children are present, while Americans speed up. In both

287 samples, children were carried at significantly different speeds than similarly-sized loads of food 288 or goods.

It is worth noting that our adult walking speeds are slower than some speeds previously recorded in the western contexts (see (42) for review of speed data from across 10 studies).

291 Substantial ethnographic data, however, show speeds similar to those we found here, particularly

292 among people walking in groups carrying loads typical of those seen here. Female !Kung (43)

293 and Xhosa (44) foragers, for instance, both walked around $0.9 \mathrm{~m} / \mathrm{s}$, Hadza women walked at 0.97

$294 \mathrm{~m} / \mathrm{s}(45)$, and Ache women walked at $0.8 \mathrm{~m} / \mathrm{s}(46)$. We believe that our data are more

295 comparable to the foraging data, rather than to other published urban data due to our location-- 
296 which is oriented around a common goal or 'function' of the walking behavior — that is, in our

297 sample, people were walking towards or away from a market/gathering area which is similar to

298 the goal and location of typical speed snapshots of foragers. We did not collect data across a

299 cross walk, nor in a central downtown area in which people are highly compacted and moving to

300 and from work and/or lunch breaks. These are the locations typical of many speed-related studies

301 on urban populations and most likely represent more rapid walking. In other work we have also

302 collected data on people crossing streets, and these data show people walking an average speed

303 of $1.4 \mathrm{~m} / \mathrm{s}$, though loaded people walk more slowly than this (47). Further studies comparing and

304 contrasting walking speed in the same city at different intersections will be an obvious next step

305 for this research.

306 Our data also show that American walkers in groups walk faster than those walking

307 alone. Though pedestrians generally slowdown in groups $(9,12,48,49)$, some data do indicate

308 that individual and paired walkers walk at equivalent speeds, though this is clearly situationally

309 dependent (39). Our findings highlight the specific nature of our "errand-running" observational

310 setting; larger groups may additionally be associated with higher task demand and hurriedness in

311 this naturalistic setting.

312 Selecting a speed for a particular walking task involves a complex set of interactive

313 variables that include both physiological (metabolic energy expended, heat load, and water loss)

314 as well as behavioral (time spent on the task and the possibility of socialization) variables $(8,9$,

315 50). Because of the habitual nature of walking, small differences in these costs will accumulate

316 into the large changes that over time that influence fitness (51). Speed adjustment decisions,

317 then, are expected to be the product of selective tradeoffs for minimizing costs and

318 thermoregulatory burdens, while maximizing task accomplishments $(8,52)$. While previous 
319 studies by Bornstein and Bornstein (32) and Levine and Norenzayan (53) compare walking

320 speeds across 6 and 31 countries, respectively in a variety of contexts, we provide higher

321 resolution data demonstrating cultural variation in the driving forces behind walking behaviors.

322 Ugandans in groups, for example, accept a higher time cost by selecting slower speeds in

323 groups than alone. This strategy has been widely observed in ecological $(12,49)$ and controlled

324 walking studies $(9,48)$. Two main reasons have been suggested as to why people choose to slow

325 down when walking together. First, differences in optimum walking speed based on size and

326 sexual dimorphism generally lead to faster group members deviating from their energetically

327 optimal speed to accommodate slower walkers in the group (12). Second, slower walking speeds

328 are correlated to closer interpersonal distances between walkers, such that any increased costs

329 from walking more slowly may be outweighed by the benefit of social investment and bonding

$330(9,12,54)$. Faster walkers are less aligned with their walking partners (i.e. they are more

331 staggered), so interpersonal contact may have to be sacrificed to walk quickly. Our data for

332 Ugandan mixed sex groups, groups of women, and groups with children are consistent with these

333 two explanations. Our finding, though, that Ugandan men walk more slowly with other men than

334 when alone challenges an existing framework for men. It has been accepted that many Western

335 men speed up when walking with other men due to a high societal emphasis on hierarchy and

336 competition among males $(9,12,14)$. A difference in male-male relationship dynamics would

337 explain the slower walking speeds, and potentially psychological closeness of Ugandan men.

338 Gendered relationship norms also clearly vary across cultures. These norms prescribe

339 appropriate interactions between genders, including interpersonal distances. Baxter (55), for

340 instance, finds that interpersonal distances of same- and mixed-sex pairs differed based on

341 ethnicity in the United States. Traditionally gendered traits, such as competitiveness, have also 
342 been shown to vary culturally. Amongst the matrilineal Khasi, for example, women show higher

343 competitiveness than men, while men tend toward competitiveness in patrilineal cultures (56).

344 The Baganda, Central Uganda's predominant ethnic group, are a patrilineal group like most

345 Western cultures (57); however they are a high-contact culture $(58,59)$. High-contact cultures,

346 like the Baganda, have been shown to interact at closer distances than people from low-contact

347 cultures like the United States (60). Uganda's numerous additional ethnic groups similarly

348 follow high-contact tendencies. While residents of the Northwestern United States originate from

349 diverse ethnic groups of both low- and high-contact natures, American cultural norms align with

350 low-contact values. Tendency toward closer interpersonal contact may explain why all Ugandan

351 walking groups, including groups of men, slow down when walking together--because slowing

352 down leads to closer contact between walkers (12).

353 We also find that, in both our Ugandan and United States samples, children are carried at

354 significantly different speeds than other load types. That children are transported differently than 355 other loads is widely recognized $(19,61)$. Hodges and Lindhiem (62) show that walkers carrying 356 children are perceived as more cautious than those carrying groceries, regardless of their actual

357 gait. Infant carrying emerged early in the primate lineage, whereas foraging-related burden

358 carrying has been thought to emerge in early members of the genus $\operatorname{Homo}(24,25,63)$. We can

359 expect then, that these two types of load-carrying evolved under different constraints. Our data

360 show that children are carried at faster speeds than other loads in the North American sample,

361 but at slower speeds in the Ugandan sample. Kramer (61) predicts that mothers should carry their

362 infants--rather than allow them to walk independently--when the carrying mother's energy

363 expenditure is less than that of the independently walking pair. At faster speeds, she argues, it is

364 beneficial to carry the child. Our data, however, show that groups with individuals carrying 
365 children walk at similar speeds to groups with children independently walking. Additionally,

366 Ugandans also carry children at slower speeds (and not at faster) which seems to conflict with

367 Kramer's model (61). Either Ugandan's choices are influenced by parental care norms not

368 addressed in Kramer's model, or the nature of Ugandans' walking task differs from Americans'

369 and those predicted by Kramer's model. As discussed above, interpersonal contact is more

370 normative in Baganda culture than in Western cultures. Infants in African groups such as the

371 !Kung or Gusii are held or touched about $70-80 \%$ of daylight hours, compared to $12-20 \%$ in

372 industrialized nations (64). Higher contact norms unaccounted for in Kramer's efficiency model

373 (61) may impact the decision to carry a child, even at slower speeds. It is also possible that

374 Ugandans choose to carry at slower speeds because their errand-running task requires walking

375 longer distances. In this case, it is energetically favorable to incur child-carrying costs because

376 allowing a child to walk over long distances will accumulate into greater total energy expended

377 for the pair in addition to the larger time-costs considering the lower optimal walking speeds of

378 children $(65,66)$.

379

380 CONCLUSION

381 The differences in the walking speeds of groups and when carrying children between

382 Central Ugandan and Northwest United States samples accentuates the role of culture and

383 environment in mediating decisions that have energetic consequences. In the future, greater

384 efforts should be taken to understand the ways in which walking behaviors vary across cultures

385 (see (67)). Our data also show that existing predictions of how humans will respond to locomotor

386 or mobility challenges are specific to one sample and may not generalize across cultural groups.

387 It is also important to recognize the ways in which behavioral differences between groups, either 
388 between sexes or across populations, are influenced by external social factors such as dominance

389 structures in addition to innate biological or morphological differences (e.g. (68); see (69)). Such

390 recognition builds an understanding that behaviors are environmentally situated processes rather

391 than static attributes.

392

393 ACKNOWLEDGEMENTS

394

395 We offer many thanks to A. Belleville, B.R. Apouli, and B.A. Asiimwe for their assistance in

396 data collection and to A. Luthi for her help with data entry. K. Neuhouser and M.J. Myers gave

397 helpful comments that have greatly improved the paper. 
398 Figure Headings

399

400

401

402

403

404

405

406

407

408

409

410

411

412

413

414

415

416

417

418

419

420

421
Fig. 1: Average speeds of Ugandans (dark grey) and Americans (light grey) based on walking group size (x-axis refers to number of people walking in the group). Error bars indicate 95\% CI.

Fig.2: Average speeds of Ugandans (dark grey) and Americans (light grey) carrying either children, food, or goods. Error bars indicate $95 \%$ CI. The solid black line represents the unloaded mean for Ugandans; the dotted grey line represents the unloaded mean for Americans.

Fig. 3: Average speeds of Ugandans (dark grey) and Americans (light grey) based on walking group composition. Both group composition and location had a significant impact on speed $(p<0.001 ; p=0.036)$, and significantly interacted $(p<0.001)$. The dashed lines demarcate Tukey's post-hoc results from our GLM for Ugandan walkers - the speeds in the bracket adjacent to the single walkers were not significantly different from them. For American walkers, only children walking together had walking speeds similar to adults walking alone (American adults walked more slowly when walking alone; children together were similar to this).Error bars indicate $95 \%$ CI. The confidence intervals for the walkers without a group are so small they are covered by the dot itself. 
423 References

424

425

426

427

428

429

430

431

432

433

434

435

436

437

438

439

440

441

442

443

444

445

446

447

448

449

450

451

452

453

454

455

456

457

458

459

460

461

462

463

464

465

466

467

468

469

470

471

1. Bertram JE \& Ruina A (2001) Multiple walking speed-frequency relations are predicted by constrained optimization. J. Theor. Biol. 209(4):445-453.

2. Ralston HJ (1958) Energy-speed relation and optimal speed during level walking. Eur. J. Appl. Physiol. 17:277-283.

3. Selinger JC, O'Connor JD, Wong JD, \& Donelan JM (2015) Humans can continuously optimize energetic cost during walking. Curr. Biol. 25:2452-2456.

4. Srinivasan M \& Ruina A (2006) Computer optimization of a minimal biped model discovers walking and running. Nature 439(7072):72-75.

5. Zarrugh MY, Todd FN, \& Ralston HJ (1974) Optimization of energy expenditure during level walking. Eur. J. Appl. Physiol. 33(4):293-306.

6. Torrence R (1989) Retooling: towards a behavioral theory of stone tools. Time, Energy and Stone Tools, ed Torrence R (Cambridge University Press, Cambridge), pp 57-66.

7. Pollard KA \& Blumstein DT (2008) Time allocation and the evolution of group size. Anim. Behav. 76:1683-1699.

8. Wall-Scheffler CM \& Myers MJ (2013) Reproductive costs for everyone: How female loads impact human mobility strategies. J. Hum. Evol. 64(5):448-456.

9. Wagnild JM \& Wall-Scheffler CM (2013) Energetic consequences of human sociality: Walking speed choices among friendly dyads. PLoS ONE 8(10):e76576.

10. Pontzer $\mathrm{H}$ (2007) Limb length and the scaling of locomotor cost in terrestrial animals. J. Exp. Biol. 210:1752-1761.

11. Steudel K (1994) Locomotor energetics and hominid evolution. Evol. Anthropol. 3:42-48.

12. Costa M (2010) Interpersonal distances in group walking. Journal of Nonverbal Behavior 34:15-26.

13. Wall-Scheffler CM (2012) Size and shape: Morphology's impact on human speed and mobility. J. Anthropol. 2012:1-9.

14. Boles W (1981) The effect of density, sex and group size upon pedestrian walking velocity. Man-Environment Systems 11(1-2):37-40.

15. Bastien GJ, Willems PA, Schepens B, \& Heglund NC (2005) Effect of load and speed on the energetic cost of human walking. Eur. J. Appl. Physiol. 94(1-2):76-83.

16. Wall-Scheffler CM, Wagnild JM, \& Wagler E (2015) Human footprint variation while performing load bearing tasks. PLoS ONE 10(3):e0118619.

17. Bertram JE (2005) Constrained optimization in human walking: Cost minimization and gait plasticity. J. Exp. Biol. 208:979-991.

18. DiPrampero PE (1986) The energy cost of human locomotion on land and in water. Int. J. Sports Med. 7:55-72.

19. Kramer PA (2004) Burden transport: When, how and how much? Socioeconomic Aspects of Human Behavioral Ecology: Research in Economic Anthropology 23:247267.

20. Maloiy GM, Heglund NC, Prager LM, Cavagna GA, \& Taylor CR (1986) Energetic cost of carrying loads: Have African women discovered an economic way? Nature 319(6055):668-669.

21. Abe D, Yanagawa K, \& Niihata S (2004) Effects of load carriage, load position and walking speed on energy cost of walking. Appl. Ergon. 35:329-335.

22. Watson JC, Payne RC, Chamberlain AT, Jones RK, \& Sellers WI (2008) The energetic costs of load-carrying and the evolution of bipedalism. J. Hum. Evol. 54(5):675-683. 
472 23. Panter-Brick C (1992) The energy cost of common tasks in rural Nepal: Levels of energy

473

474

475

476

477

478

479

480

481

482

483

484

485

486

487

488

489

490

491

492

493

494

495

496

497

498

499

500

501

502

503

504

505

506

507

508

509

510

511

512

513

514

515

516

517

518

519

520

521

522 expenditure compatible with sustained physical activity. Eur. J. Appl. Physiol. 64:477484.

24. Ross C (2001) Park or ride? Evolution of infant carrying in primates. Int. J. Primatol. 22(5):749-771.

25. Rhinegold HL \& Keen GC (1963) Transport of the human young. Determinants of Infant Behavior III, ed Foss BM (Methuen \& Co. Ltd., London), pp 87-110.

26. Sallstrom J, Snyder KL, \& Wall-Scheffler CM (2012) Baby on the hip: How do urban individuals carry infants? in American Association of Physical Anthropology (Portland, OR, USA).

27. Burgess AP \& Burgess HJL (1964) The growth pattern of East African schoolgirls. Hum. Biol. 36(2):177-193.

28. Fryar CD, Gu Q, Ogden CL, \& Flegal KM (2016) Anthropometric reference data for children and adults: United States, 2011-2014. in Vital Health Statistics (National Center for Health Statistics).

29. Amato P (1983) The effects of urbanization on interpersonal behavior: Field studies in Papua New Guinea. Journal of Cross-Cultural Psychology 14(3):353-367.

30. Arango J \& Montufar J (2008) Walking speed of older pedestrians who use canes or walkers for mobility. Transportation Research Record 2073(79-85).

31. Bohannon RW (1997) Comfortable and maximum walking speed of adults aged 20-79 years: reference values and determinants. Age Ageing 26:15-19.

32. Bornstein MH \& Bornstein HG (1976) The pace of life. Nature 259(5544):557-559.

33. Coffin A \& Morrall J (1995) Walking speeds of elderly pedestrians at crosswalks. Transportation Research Record (1487):63-67.

34. Elman D, Schulte D, \& Bukoff A (1977) Effects of facial expression and stare duration on walking speed: Two field experiments. Journal of Nonverbal Behavior 2(2):93-99.

35. Gates TJ, Noyce DA, Bill AR, \& Van Ee N (2006) Recommended walking speeds for timing of pedestrian clearance intervals based on characteristics of the pedestrian population. Transportation Research Record 1982:38-47.

36. Knoblauch R, Peitrucha M, \& Nitzburg M (1996) Field studies of pedestrian walking speed and start-up time. Transportation Research Record 1538:27-38.

37. Korte C \& Grant R (1980) Traffic noise, envrionmental awareness, and pedestrian behavior. Environment and Behavior 12(3):408-420.

38. Koushki PA (1988) Walking characteristics in Central Riydah, Saudi Arabia. Journal of Transportation Engineering 114(6):735-744.

39. Tarawneh MS (2001) Evaluation of pedestrian speed in Jordan with investigation of some contributing factors. Journal of Safety Research 32(2):229-236.

40. Alcantara R \& Wall-Scheffler CM (2017) Stroller running: Energetic and kinematic changes across pushing methods. PLoS ONE 12(7):e0180575.

41. Zivotofsky AZ \& Hausdorff JM (2007) The sensory feedback mechanisms enabling couples to walk synchronously: An initial investigation. Journal of NeuroEngineering and Rehabilitation 2007(4):28.

42. Ishaque MM \& Noland RB (2008) Behavioural issues in pedestrian speed choice and street crossing behavior: A review. Transport Reviews 28(1):61-85.

43. Bentley GR (1985) Hunter-gatherer energetics and fertility: A reassessment of the !Kung San. Hum. Ecol. 13:79-109.

44. Lloyd R, Parr B, Davies S, \& Cooke C (2010) Subjective perceptions of load carriage on the head and back in Xhosa women. Appl. Ergon. 41:522-529.

45. Marlowe FW (2006) Central place provisioning: The Hadza as an example. Feeding Ecology in Apes and Other Primates, eds Hohmann G, Robbins M, \& Boesch C (Cambridge University Press, Cambridge), pp 359-377. 
523

524

525

526

527

528

529

530

531

532

533

534

535

536

537

538

539

540

541

542

543

544

545

546

547

548

549

550

551

552

553

554

555

556

557

558

559

560

561

562

563

564

565

566

567

568

569

570

571

572

573

46. Hurtado AM, Hawkes K, Hill K, \& Kaplan H (1985) Female subsistence strategies among Ache hunter-gatherers of Eastern Paraguay. Hum. Ecol. 13(1):1-28.

47. Bonner-Harris A, Stohler E, Lee N, Wilson T, \& Wall-Scheffler CM (2018) Human walking behavior at crosswalks. in University of Washington Undergraduate Research Symposium (Mary Gates Hall, Seattle, WA). https://tinyurl.com/y9c7hjb8

48. Frimenko R, Goodyear C, \& Bruening D (2016) Changes in spatiotemporal differences between the sexes due to paired walking. (INFOSCITEX CORP DAYTON OH), p 14.

49. Moussaid M, Perozo N, Garnier S, Helbing D, \& Theraulaz G (2010) The walking behaviour of pedestrian social groups and its impact on crowd dynamics. PLoS ONE 5(4):e10047.

50. Wall-Scheffler CM \& Myers MJ (2017) The biomechanical and energetic advantages of a mediolaterally wide pelvis in women. The Anatomical Record 300(4):764-775.

51. Gibson MA \& Mace R (2006) An energy-saving development initiative increases birth rate and childhood malnutrition in rural Ethiopia. PLoS Medicine 3(4):476-484.

52. Miller RH, Umberger BR, Hamill J, \& Caldwell GE (2012) Evaluation of the minimum energy hypothesis and other potential optimality criteria for human running. Proc. $R$. Soc. Lond. B. Biol. Sci. doi:10.1098.

53. Levine RV \& Norenzayan A (1999) The pace of life in 31 countries. Journal of CrossCultural Psychology 30:178-205.

54. Wellens AR \& Goldberg ML (1978) The effects of interpersonal distance and orientation upon the perception of social relationships. J. Psychol. 99:39-48.

55. Baxter JC (1970) Interpersonal spacing in natural settings. Sociometry 33(4):444-456.

56. Gneezy U, Leonard K, \& List J (2009) Gender differences in competition: Evidence from a matrilineal and a patriarchal society. Econometrica 77:1637-1664.

57. Wyrod R (2008) Between women's rights and men's authority: Masculinity and shifting discources of gender difference in urban Uganda. Gender and Society 22:799-823.

58. Awa NE (1988) Communication in Africa: Implications for development planning. Howard Journal of Communication 1(3):131-144.

59. Watson OM (1970) Proxemic Behavior: A Cross-Cultural Study (Walter de Gruyter).

60. Remland MS, Jones TS, \& Brinkman H (1995) Interpersonal distance, body orientation, and touch: Effects of culture, gender, and age. J. Soc. Psychol. 135:281-297.

61. Kramer PA (1998) The costs of human locomotion: Maternal investment in child transport. Am. J. Phys. Anthropol. 107:71-85.

62. Hodges BH \& Lindhiem O (2006) Carrying babies and groceries: The effect of moral and social weight on caring. Ecological Psychology 18:93-111.

63. Leonard WR \& Robertson ML (1997) Comparative primate energetics and hominid evolution. Am. J. Phys. Anthropol. 102:265-281.

64. Hewlett BS (1996) Diverse contexts of human infancy. Cross-Cultural Research for Social Science, eds Ember CR \& Ember M (Prentice Hall, Upper Saddle River, NI), pp 330.

65. Cavagna GA, Franzetti AP, \& Fuchimoto T (1983) The mechanics of walking in children. J. Physiol. 343(1):323-339.

66. DeJaeger D, Willems PA, \& Heglund NC (2001) The energy cost of walking in children. Pflügers Arch. Eur. J. Physiol. 441:538-543.

67. Bornstein MH (2002) Toward a multiculture, multiage, multimethod science. Hum. Dev. 45(4):257-263.

68. Travis CB \& Yeager CP (1991) Sexual selection, parental investment, and sexism. Journal of Social Issues 47(3):117-129.

69. Dingwall HL, Hatala KG, Wunderlich RE, \& Richmond BG (2013) Hominin stature, body mass, and walking speed estimates based on 1.5 million-year-old footprints at lleret, Kenya. J. Hum. Evol. 64(6):556-568. 


\section{Figure 1}

Average speeds of Ugandans (dark grey) and Americans (light grey) based on walking group size ( $x$-axis refers to number of people walking in the group).

Error bars indicate $95 \% \mathrm{Cl}$.

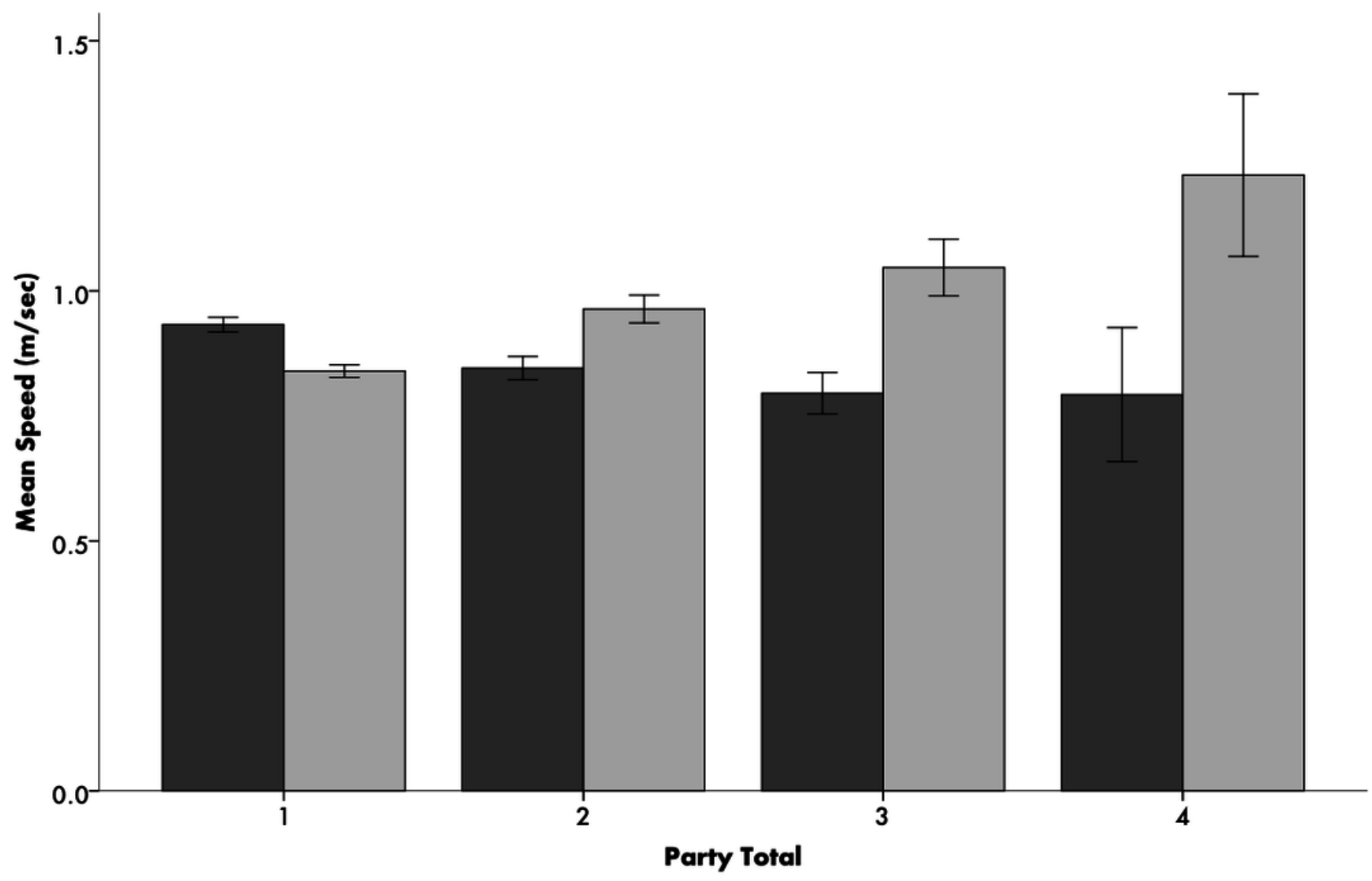




\section{Figure 2}

Average speeds of Ugandans (dark grey) and Americans (light grey) carrying either children, food, or goods.

Error bars indicate $95 \% \mathrm{Cl}$. The solid black line represents the unloaded mean for Ugandans; the dotted grey line represents the unloaded mean for Americans.

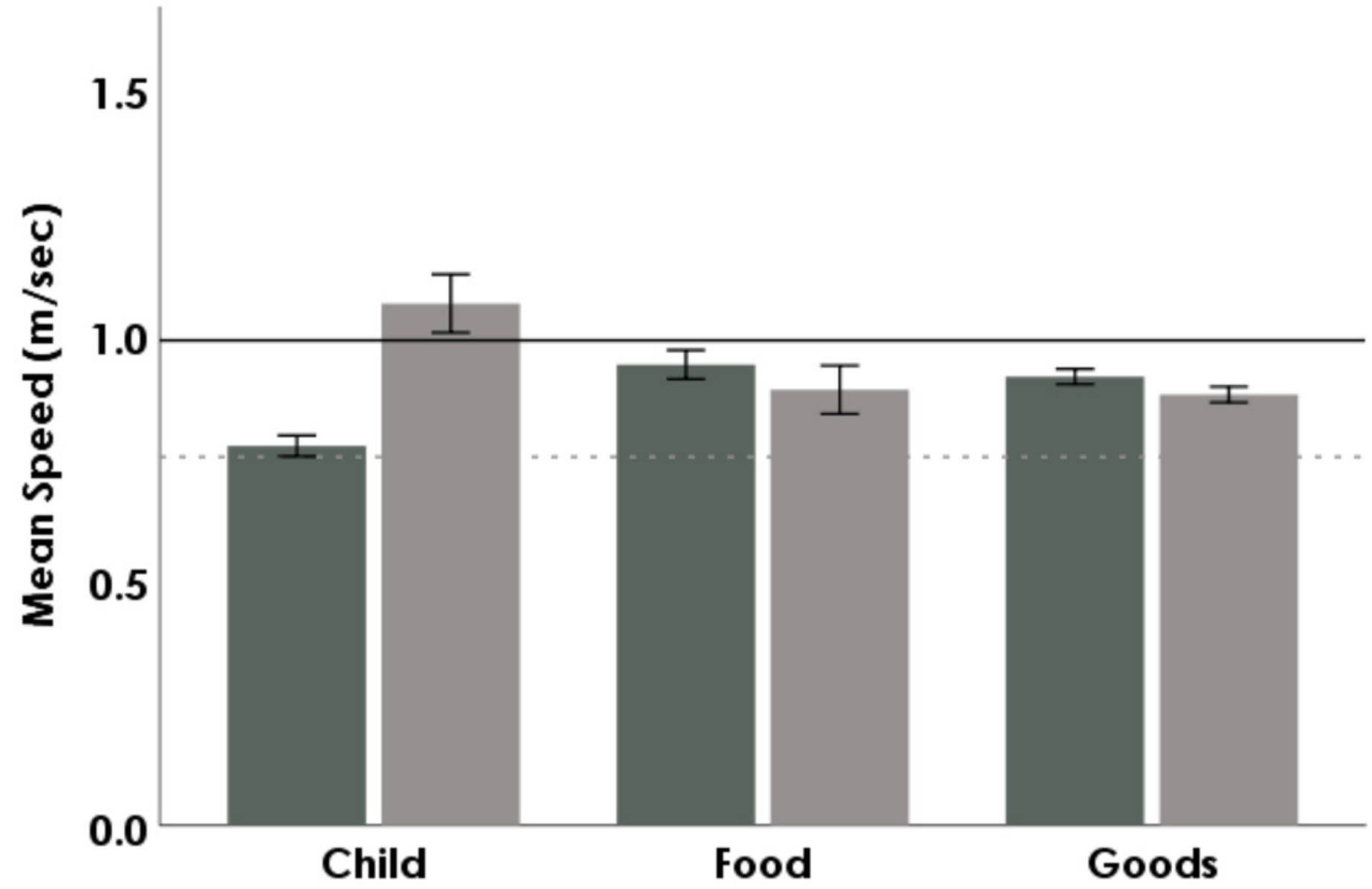




\section{Figure 3}

Average speeds of Ugandans (dark grey) and Americans (light grey) based on walking group composition.

Both group composition and location had a significant impact on speed $(p<0.001 ; p=0.036)$, and significantly interacted $(p<0.001)$. The dashed lines demarcate Tukey's post-hoc results from our GLM for Ugandan walkers-the speeds in the bracket adjacent to the single walkers were not significantly different from them. For American walkers, only children walking together had walking speeds similar to adults walking alone (American adults walked more slowly when walking alone; children together were similar to this). Error bars indicate $95 \% \mathrm{Cl}$. The confidence intervals for the walkers without a group are so small they are covered by the dot itself. 


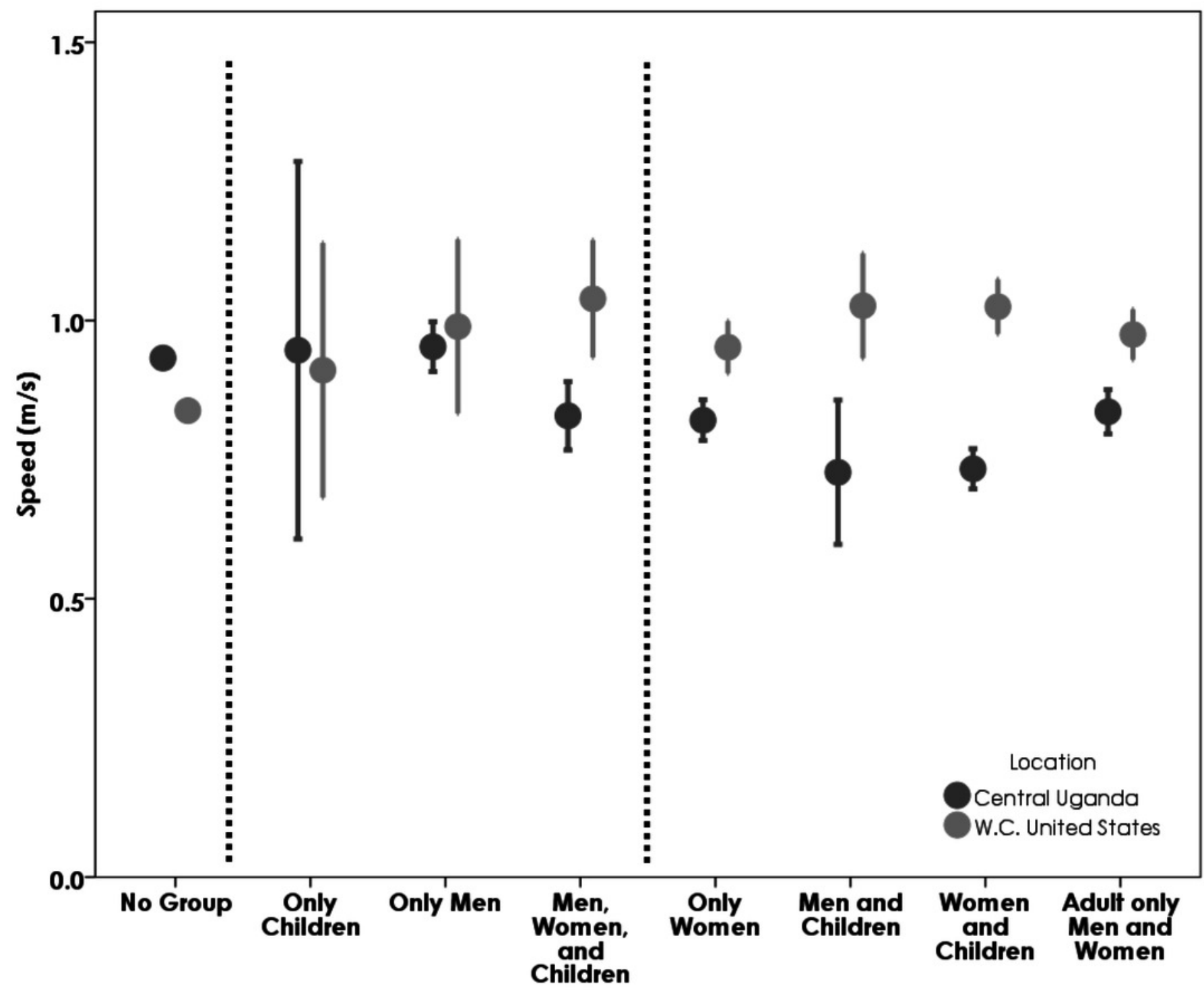

Error Bars: 95\% Cl 


\section{Table $\mathbf{1}$ (on next page)}

Focal subject age categorization guidelines.

Average height measurements calculated as the average height of American males and females in that designated age group according to the 2016 CDC Anthropomorphic Reference (Fryar, Gu, Ogdern, and Flegal 2016). 
1 Table 1: Focal subject age categorization guidelines. Average height measurements calculated 2 as the average height of American males and females in that designated age group according to 3 the 2016 CDC Anthropomorphic Reference (Fryar, Gu, Ogdern, and Flegal 2016).

4

\begin{tabular}{|l|l|}
\hline Child & $\begin{array}{l}\text { Estimated age 3-12 years; immature facial features, } \\
\text { short stature, and commonly accompanied by older } \\
\text { persons }\end{array}$ \\
\hline Teenager & $\begin{array}{l}\text { Estimated age 13-18 years; average height 1.67 } \\
\text { meters; older school-aged individuals, often } \\
\text { carrying backpacks }\end{array}$ \\
\hline Adult & \begin{tabular}{l} 
Estimated age 19-59 years \\
\hline Older \\
Adult
\end{tabular} \\
\hline $\begin{array}{l}\text { Estimated age 60+; characterized by greying hair, } \\
\text { developed wrinkles, and/or stooped posture }\end{array}$ \\
\hline
\end{tabular}




\section{Table 2 (on next page)}

Load categorization guidelines with example load descriptions and masses for loads of each size and type category.

Child mass measurements calculated as the average mass of American males and females in that designated age group according to the 2016 CDC Anthropomorphic Reference (Fryar, Gu, Ogdern, and Flegal 2016). 
1 Table 2: Load categorization guidelines with example load descriptions and masses for loads of 2 each size and type category. Child mass measurements calculated as the average mass of

3 American males and females in that designated age group according to the 2016 CDC

4 Anthropomorphic Reference (Fryar, Gu, Ogdern, and Flegal 2016).

5

\begin{tabular}{|l|l|l|l|}
\hline Load Size & Type & $\begin{array}{l}\text { Description } \\
\text { Coffee cup or beverage; } \\
\text { individual snack food items } \\
\text { able to be handled with one } \\
\text { hand. }\end{array}$ & $<1 \mathrm{~kg}$ \\
\hline Food & Goods & $\begin{array}{l}\text { Thin books, paper folders, } \\
\text { clutch-sized purse }\end{array}$ & $<1 \mathrm{~kg}$ \\
\hline Medium & Food & $\begin{array}{l}\text { A single, full bag of groceries, } \\
\text { bundle of food for sale } \\
\text { approximately of torso size; } \\
\text { visibly full }\end{array}$ & $1-6 \mathrm{~kg}$ \\
\hline Goods & $\begin{array}{l}\text { Bag or mid-sized purse } \\
\text { approximately of torso size } \\
\text { Child }\end{array}$ & $\begin{array}{l}\text { Infant (unable to mobilize } \\
\text { independently) }\end{array}$ & $\bar{x} \approx 6.5 \mathrm{~kg}$ \\
\hline Food & $\begin{array}{l}\text { Packages of groceries or food } \\
\text { for sale larger than torso-sized }\end{array}$ & $>6 \mathrm{~kg}$ \\
\hline Goods & $\begin{array}{l}\text { Oversized bag (larger than } \\
\text { torso size) }\end{array}$ & $>6 \mathrm{~kg}$ \\
\hline Child & $\begin{array}{l}\text { Toddler or Child (able to } \\
\text { mobilize independently) }\end{array}$ & $11.5-19.7 \mathrm{~kg}$ \\
\hline Stroller & $\begin{array}{l}\text { Child-carrying device pushed } \\
\text { in front of walker }\end{array}$ & $\begin{array}{l}13 \mathrm{~kg} \text { (stroller } \\
\text { alone) plus } 6-15 \mathrm{~kg} \\
\text { (size of child) }\end{array}$ \\
\hline
\end{tabular}




\section{Table 3(on next page)}

Count and mean, minimum, and maximum speeds of walkers in Central Uganda and West Coast United States.

No subjects walked with strollers in the Central Ugandan population. 


\begin{tabular}{|c|c|c|c|c|c|c|c|c|c|c|}
\hline & & & \multicolumn{4}{|c|}{ Central Uganda } & \multicolumn{4}{|c|}{ W.C. United States } \\
\hline & & & Count & $\begin{array}{c}\text { Mean Speed } \\
(\mathrm{m} / \mathrm{s})\end{array}$ & Min. & Max. & Count & $\begin{array}{c}\text { Mean Speed } \\
(\mathbf{m} / \mathbf{s})\end{array}$ & Min. & Max. \\
\hline \multirow{2}{*}{\multicolumn{2}{|c|}{$\begin{array}{c}\text { Unloaded Adults } \\
\text { Alone }\end{array}$}} & Male & 40 & 1.04 & 0.61 & 1.59 & 108 & 0.8 & 0.58 & 1.23 \\
\hline & & Female & 14 & 0.89 & 0.71 & 1.22 & 37 & 0.84 & 0.56 & 1.23 \\
\hline \multirow{4}{*}{$\begin{array}{l}\text { Load } \\
\text { Condition }\end{array}$} & \multirow{2}{*}{ Unloaded } & Male & 48 & 1.02 & 1.59 & 0.53 & 143 & 0.84 & 1.44 & 0.57 \\
\hline & & Female & 16 & 0.90 & 1.22 & 0.71 & 64 & 0.90 & 1.29 & 0.56 \\
\hline & \multirow{2}{*}{ Loaded } & Male & 307 & 1.00 & 1.69 & 0.45 & 194 & 0.87 & 1.66 & 0.53 \\
\hline & & Female & 598 & 0.84 & 1.44 & 0.38 & 351 & 0.89 & 1.66 & 0.54 \\
\hline \multirow{8}{*}{$\begin{array}{l}\text { Load } \\
\text { Type }\end{array}$} & \multirow{2}{*}{ Child } & Male & 11 & 0.81 & 1.00 & 0.62 & 4 & 1.06 & 1.28 & 0.96 \\
\hline & & Female & 132 & 0.77 & 1.08 & 0.38 & 11 & 1.06 & 1.21 & 0.89 \\
\hline & \multirow{2}{*}{ Food } & Male & 69 & 1.01 & 1.36 & 0.62 & 15 & 0.85 & 1.12 & 0.71 \\
\hline & & Female & 90 & 0.88 & 1.44 & 0.47 & 37 & 0.90 & 1.45 & 0.66 \\
\hline & \multirow{2}{*}{ Goods } & Male & 227 & 1.00 & 1.69 & 0.45 & 171 & 0.87 & 1.66 & 0.53 \\
\hline & & Female & 376 & 0.86 & 1.38 & 0.46 & 287 & 0.88 & 1.66 & 0.54 \\
\hline & \multirow{2}{*}{ Stroller } & Male & 0 & & & & 4 & 0.99 & 1.14 & 0.81 \\
\hline & & Female & 0 & & & & 16 & 0.95 & 1.16 & 0.77 \\
\hline $\begin{array}{l}\text { Subject } \\
\text { Papty }\end{array}$ & Alone & Male & 258 & 1.03 & 1.69 & 0.53 & 273 & 0.83 & 1.56 & 0.53 \\
\hline
\end{tabular}

1 Party fable 3: Count and mean, minimum, and maximum speeds of walkers in Central Uganda and West Coast United States. No subjeets

2 walked with strollers in the Central Ugandan population. 


\begin{tabular}{|c|c|c|c|c|c|c|c|c|c|c|}
\hline & & Female & 405 & 0.87 & 1.38 & 0.38 & 278 & 0.84 & 1.25 & 0.54 \\
\hline & & Male & 97 & 0.91 & 1.54 & 0.45 & 65 & 0.96 & 1.66 & 0.63 \\
\hline & Group & Female & 209 & 0.80 & 1.44 & 0.43 & 136 & 1.00 & 1.66 & 0.61 \\
\hline \multirow{4}{*}{$\begin{array}{l}\text { Children } \\
\text { Present }\end{array}$} & \multirow{2}{*}{$\begin{array}{l}\text { No } \\
\text { Children }\end{array}$} & Male & 329 & 1.01 & 1.69 & 0.53 & 303 & 0.85 & 1.66 & 0.53 \\
\hline & & Female & 432 & 0.87 & 1.44 & 0.46 & 351 & 0.87 & 1.66 & 0.54 \\
\hline & \multirow{2}{*}{$\begin{array}{l}\text { Children } \\
\text { Present }\end{array}$} & Male & 26 & 0.82 & 1.54 & 0.45 & 35 & 0.98 & 1.33 & 0.66 \\
\hline & & Female & 182 & 0.78 & 1.21 & 0.38 & 63 & 1.01 & 1.39 & 0.74 \\
\hline
\end{tabular}

